\title{
Evaluation of Serum Paraoxonase Levels and Dyslipidemia in Chronic Urticaria Patients
}

\author{
A.A.Ibrahim ${ }^{1}$, A.M.Hamed ${ }^{1}$, A.A.Hafez ${ }^{2}$ and M.M.Aref ${ }^{3}$ \\ ${ }^{1}$ Dermatology, Venereology and Andrology Dept., Faculty of Medicine, Benha Univ., Benha, Egypt \\ ${ }^{2}$ Clinical and Chemical Pathology Dept., Faculty of Medicine, Benha Univ., Benha, Egypt \\ ${ }^{3}$ Dermatology,Venereology and Andrology Dept.,Tanta Dermatology and Leprosy Hospital , Gharbeia , Egypt \\ E.Mail: Mahetab82@gmail.com
}

\begin{abstract}
Constant urticaria (CU) is an upsetting unfavorably susceptible state of the skin. The urticarial injuries are available persistently or irregularly for at any rate a month and a half. The sores are edematous pruritic pink wheals of variable size and shape, with or without going with angioedema. It has high effect on rest and daytime exercises including work, or potentially inadequate reactions to standard treatment. Notwithstanding illness seriousness, the unconventionality of $\mathrm{CU}$ and its course are a significant reason for worry for patients. Persistent urticaria is viewed as a constant incendiary illness as portrayed by cutaneous pole cell degranulation, and invading $\mathrm{T}$ cells, eosinophils, and neutrophils. Expanded circling levels of C-receptive protein (CRP) and proinflammatory cytokines, for example, interleukin (IL)- 6 , tumor rot factor (TNF)- $\alpha$, and network metalloproteinase (MMP)- 9 were seen among patients with CU. A fundamental favorable to provocative and supportive of coagulating state, shown by raised degrees of incendiary markers, for example, IL-1, IL-6, TNF, and CRP, was accounted for among people with hyperlipidemia. Serum paraoxonase (PON) is a high thickness lipoprotein (HDL) bound compound displaying antiatherogenic properties.This chemical assumes a significant function in forestalling low thickness lipoprotein (LDL) oxidation and is considered to secure against the advancement of coronary illness. Point: The point of this examination is to asses serum paraoxonase levels and dyslipidemia in ongoing urticaria patients and relate their levels with various factors of the illness. Technique: This current investigation included: Patient gathering (bunch 1) included 50 psoriasis patients. What's more, 30 clearly solid, age and sex coordinated people included as a benchmark (Group 2). All were explored for serum PON 1 level and analyzed. Serum PON 1 was assessed utilizing industrially accessible ELISA units. End: From the consequences of present investigation, it is presumed that serum paraoxonase levels are diminished in patients with constant urticaria contrasted with age and sex coordinated controls with correspondence to the infection seriousness.
\end{abstract}

Keywords: Urticaria, Hyperlipidemia, PON 1.

\section{Introduction}

Ongoing urticaria (CU) is an upsetting unfavorably susceptible state of the skin. The urticarial injuries are available ceaselessly or discontinuously for in any event a month and a half. Despite the fact that the predominance and qualities of $\mathrm{CU}$ are entrenched, little is thought about repetitive constant urticaria (RCU) [1].

Ongoing urticaria is an exceptionally common cutaneous illness and an incessant reason for clinical counsel. Revealed lifetime pervasiveness rates are assessed to be as high as $8-10 \%$ of the populace with a point predominance of $0.6 \%$ [2].

In this condition, the sores repeat for longer than about a month and a half as trademark hives which are edematous pruritic pink wheals of variable size and shape, with or without going with angioedema. In angioedema, the expanding is more profound than wheals and may influence mucosal surfaces. Erythema is frequently missing in angioedema, and ordinary locales of preference incorporate the eyelids, lips, and tongue [3]. In numerous patients, the infection brings about essentially disabled personal satisfaction. This is generally because of the way that $\mathrm{CU}$ - in numerous patients-is serious, with high sickness movement, the event of the two wheals and angioedema, has high effect on rest and daytime exercises including work, or potentially inadequate reactions to standard therapy. Notwithstanding infection seriousness, the unusualness of $\mathrm{CU}$ and its course are a significant reason for worry for patients [4].

Ongoing urticaria is viewed as a persistent incendiary infection as portrayed by cutaneous pole cell degranulation, and invading $\mathrm{T}$ cells, eosinophils, and neutrophils. Expanded flowing degrees of Cresponsive protein (CRP) and proinflammatory cytokines, for example, interleukin (IL)- 6, tumor rot factor (TNF)- $\alpha$, and framework metalloproteinase (MMP)- 9 were seen among patients with CU [5].

Hyperlipidemia was ensnared as being engaged with fiery cycles related with expanded dangers of resulting atherosclerosis and cardiovascular sicknesses (CVDs), and is a significant reason for death in both created and non-industrial nations [6]. A fundamental favorable to incendiary and supportive of coagulating state, represented by raised degrees of provocative markers, for example, IL-1, IL-6, TNF, and CRP, was accounted for among people with hyperlipidemia [7].

Serum paraoxonase (PON) is a high thickness lipoprotein (HDL) bound compound showing antiatherogenic properties [8]. 
This catalyst assumes a significant function in forestalling low thickness lipoprotein (LDL) oxidation and is considered to secure against the advancement of coronary illness [9].

\section{Materials and methods}

\subsection{The Study Population}

This is a case-control study .The momentum study had been directed between February 2019 and February 2020 after the endorsement by Research Committee at Faculty of Medicine, Benha University. The examination included 80 subjects that were haphazardly chosen from those going to the Dermatology Outpatient's Clinic at Benha University Hospital, Faculty of Medicine, Benha University, Qalyubia, Egypt. Preceding commencement of the examination, each subject was educated about the point of the investigation and an educated assent was acquired from every person before test assortment. This current examination included: Patient gathering (bunch 1) included 50 psoriasis patients. Moreover, 30 evidently solid, age and sex coordinated people included as a benchmark group (Group2).

\subsection{ELISA assays of PON 1}

Quantitative determinations of serum sirt1 were achieved using a double-

antibody sand wich Enzyme Linked Immune Sorbent Assay technique (ELISA) was used to detect serum level of sirt1 using a commercial Human sirt1 ELISA Kit for research use only (Cat \#: 201-122158, SunRedBio, China).

\subsection{Statistical analysis}

The collected data was revised, coded, tabulated and introduced to a PC using Statistical package for Social Science (IBM Corp. Released 2011. IBM SPSS Statistics for Windows, Version 16.0. Armonk, NY: IBM Corp.). Data were presented and suitable analysis was done according to the type of data obtained for each parameter. Student T Test was used to assess the statistical significance of the difference between two study group means. while non parametric data were presented as median and interquartile range (IQR), and analyzed by Mann Whitney $\mathrm{U}$ test (ZMWU) and Kruskal Wallis (KW) test for 2 and more than 2 independent groups respectively. Significant KW was followed by post hoc multiple comparisons using Bonferroni adjusted tests to detect the significant pairs. Non parametric correlations were assessed by Spearman's coefficient (rho). Fisher's exact test: was used to examine the relationship between two qualitative variables when the expected count is less than 5 in more than $20 \%$ of cells. Correlation analysis: To assess the strength of association between two quantitative variables. The correlation coefficient defines the strength and direction of the linear relationship between two variables. $p$ is significant if $<0.05$ at confidence interval $95 \%$.

\section{Results}

The duration of psoriasis patients in patient group ranged between 2 months to 15 years and their UAS 7 score ranged between 4-42 Table (1).

Table (1) Clinical data among group 1.

\begin{tabular}{lcc}
\hline & Rang & Mean \pm SD \\
\hline Duration & $2-180$ & $37.260 \pm 46.264$ \\
UAS 7 & $4-42$ & $23.5 \pm 9.8$ \\
Score & & \\
\hline
\end{tabular}

\subsection{Biochemical assessments of PON1}

The mean PON-1 serum levels were significantly lower in patient group than their controls Table (2) .

Table (2) Comparison between patient group and their controls regarding PON-1 (ng/dl).

\begin{tabular}{lccccc}
\hline & & Group 1 & Group 2 & Z $_{\text {MWU }}$ & P. value \\
\hline Sirt l & Range & $5.56-239.8$ & $36.9-342.1$ & 2.73 & \multirow{2}{*}{0.006} \\
$($ ng/dl) & Mean \pm SD & $96.7 \pm 36.0$ & $180.9 \pm 111.0$ & & \\
\hline
\end{tabular}

There was statistically significant negative correlation between PON-1 (ng/dl) and both TG (mg/dl) and UAS-7, but there was no statistically significant correlations between PON-1 (ng/dl) and cholesterol, BMI, age, duration of the disease Table (3).

Table (3) Correlation between serum paraoxinase and the studied variables among patients.

\begin{tabular}{lcc}
\hline \multirow{2}{*}{ With } & \multicolumn{2}{c}{ Serum paraoxonase } \\
\cline { 2 - 3 } & rho & $\mathbf{P}$ \\
\hline Age & -0.164 & 0.25 \\
Duration & 0.230 & 0.108 \\
\hline
\end{tabular}




\begin{tabular}{lcc}
\hline Table (3) Continue & & \\
\hline UAS-7 & -0.528 & $<0.001$ \\
Serum cholesterol & -0.198 & 0.16 \\
Serum TG & -0.364 & 0.009 \\
BMI & -0.117 & 0.336 \\
\hline
\end{tabular}

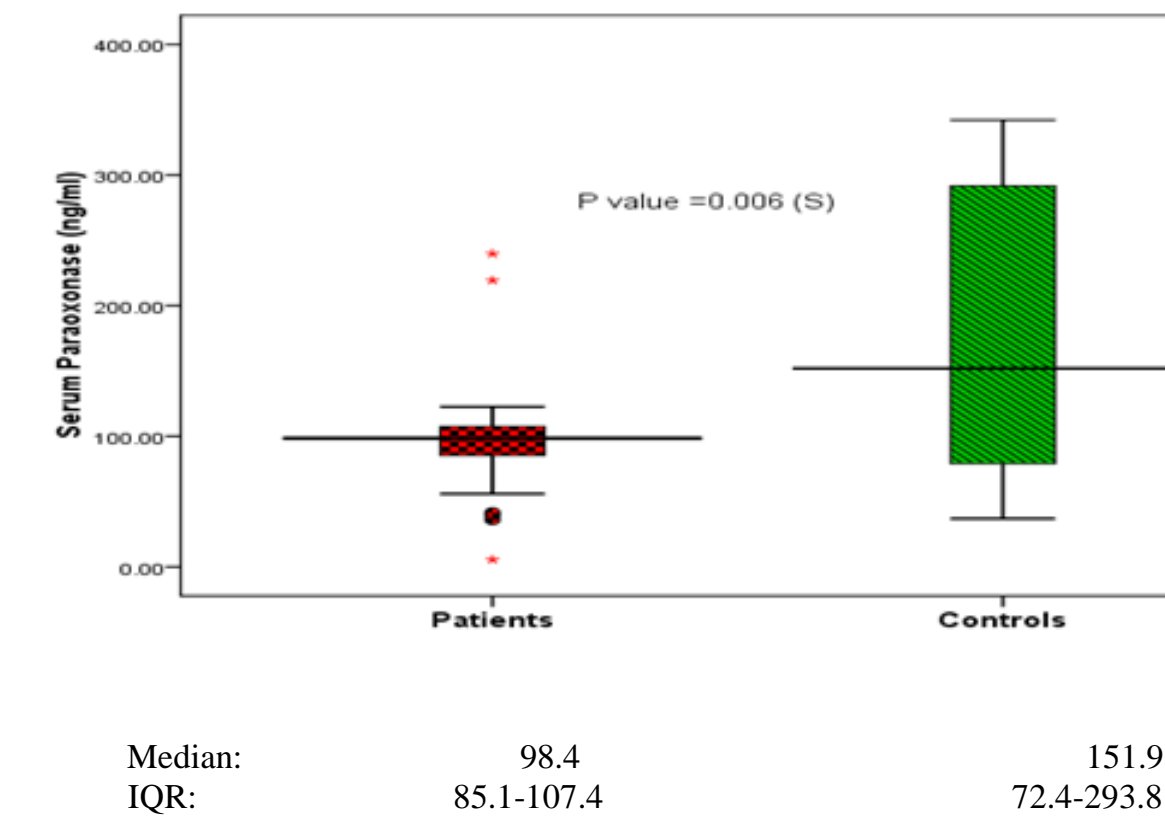

Fig (1) Box plot showing the median and IQR of serum paraoxinase among the studied groups.

\section{Discussion}

This case-controlled examination was intended to survey serum levels of PON-1 in persistent urticaria patients and connect their levels with the seriousness of the infection.

In the current investigation, there were factually critical abatement in PON-1 (ng/dl) levels among patients gathering and their controls, likewise the examination uncovered measurably huge lessening in PON-1 levels among bunch 1 than bunch 2 demonstrating that PON-1 level diminishing in ongoing urticaria.

These results can be explained as [10] Chronic urticaria is a fiery illness intervened by oxidative pressure and that pole cell and basophiles are fit for creating superoxide anion that may cause lipid peroxidation. Human serum (PON1) is [11] an esterase catalyst which has lipophilic cancer prevention agent qualities and assumes a significant function in diminishing oxidative pressure. PON1 specifically hydrolyzes supportive of incendiary oxidized lipids. [12] has revealed decreased paraoxonase exercises in patients who are under expanded oxidative pressure. Some in vitro information have emphatically proposed that expanded fatty substances decline PON1 synergist movement

In the current examination, the mean serum Triglycerides level was fundamentally higher in patients than controls, yet there was no factually critical contrast among patients and controls in the degree of serum cholesterol.

This is in correspondence with, [6] who found a 1.65-overlap expanded danger of precursor hyperlipidemia in $36.1 \%$ of $\mathrm{CU}$ patients in contrast with controls in the wake of changing for conceivable bewildering factors. Additionally [13] found a measurably huge increment of serum cholesterol, fatty substances, low thickness lipoprotein (LDL) and abatement of high thickness lipoprotein (HDL) in $\mathrm{CSU}$ in contrast with control gathering. This might be expected to [14] the huge expansion in plasma levels of TNF- $\alpha$, IL-6 and CRP in relationship with CSU clinical movement and these middle people have their functions in both irritation of urticaria and the degree of lipids in blood

In the current examination the degree of serum paraoxonase was adversely identified with the seriousness of urticaria estimated by UAS7.

Both oxidative pressure and irritation are personally connected to one another. Expansion in oxidative pressure can expand the creation of fiery cytokines and moreover, an expansion in provocative cytokines can animate the creation of free extremists [15].

It was discovered additionally that platelet oxidative pressure boundaries are fundamentally associated with plasma IL- 6 and high affectability Creceptive protein (hs-CRP) levels. This demonstrates that more serious the urticaria, more the aggravation and oxidative pressure [16]. This implies that the 
more the seriousness of urticaria, the more expansion in provocative markers which increment the oxidative pressure, making more oxidized lipids that should be deoxidized by paraoxonase, and this reduction the level and movement of PON1.

This implies that the more the seriousness of urticaria, the more expansion in fiery markers which increment the oxidative pressure, making more oxidized lipids that should be deoxidized by paraoxonase, and this diminishing the level and action of PON1.

In our investigation there was a factually huge negative connection between's serum levels of PON and serum fatty substances.

Serum PON action was demonstrated to be decreased in patients with familial hypercholesterolemia, and in patients with diabetes mellitus [17]. The capacity of PON to restrain lipoprotein oxidation, and furthermore to diminish lipoprotein-related peroxides, may speak to two separate properties of PON. To begin with, PON forestalls collection of oxidized lipid during actuated oxidation, and second, PON utilizes and in this manner wipes out preformed oxidized lipoproteins. Notwithstanding, both of these impacts may result from PON's capacity to hydrolyze explicit lipoprotein peroxides during lipoprotein oxidation [8].

The aftereffects of the current examination likewise uncovered that mental pressure $(66 \%)$ was the most widely recognized accelerating factor.

Past examinations have proposed a part for mental pressure in $\mathrm{CU}$ in a 2-way cooperation, wherein $\mathrm{CU}$ elevates mental pain and persistent pressure might be a trigger of CU. There is a proof of the event of unpleasant life functions as forerunners to the beginning of CU [18].

\section{Conclusions}

From the consequences of present investigation, it is presumed that serum paraoxonase levels are diminished in patients with persistent urticaria with correspondence to the sickness seriousness .

\section{Recommendations}

The aftereffects of our examination ought to be deciphered considering its impediments, as the current investigation incorporated a generally little example size. Further examinations are expected to research the exact instruments by which PON-1 add to the pathogenesis of both ongoing urticaria and hyperlipidemia.

\section{Conflict Of Interest}

The authors declare that they have no conflict of interest.

\section{References}

[1] J.K.Kim, D.Har, L.S.Brown and D.A.Khan , Recurrence of Chronic Urticaria: Incidence and Associated Factors. J Allergy Clin Immunol Pract,Vol.(17), PP.30537-3053, 2017.
[2] P.Gaig, M. Olona, L.D.Muñoz, M.T.Caballero, F.J.Domínguez. Epidemiology of urticaria in Spain. J Investig Allergol Clin Immunol; ,Vol.14(3), PP.214-20, 2004.

[3] W.H.Chung, C.Y.Chu, Y.H.Huang, W.M.Wang, C.H.Yang . Taiwanese Dermatological Association consensus for the definition, classification, diagnosis, and management of urticaria. J Formos Med Assoc,Vol.115(11), PP.968-980, 2016.

[4] M. Sanchez-Borges, F.Caballero-Fonseca, A.Capriles-Hulett, L.Gonzlez-Aveledo and M.Maurer . Factors linked to disease severity and time to remission in patients with chronic spontaneous urticaria. J Eur Acad Dermatol Venereol,Vol.31(6), PP.964-971, 2017.

[5] Tedeschi A, Asero R, Lorini M, Marzano AV and Cugno $M$.Plasma levels of matrix metalloproteinase-9 in chronic urticaria patients correlate with disease severity and Creactive protein but not with circulating histamine-releasing factors. Clin Exp Allergy, Vol.40, PP.875-881, 2010.

[6] Chung SD, Wang KH, Tsai MC, Lin HC, Chen CH. Hyperlipidemia Is Associated with Chronic Urticaria: A Population-Based Study. PLoS One,Vol.11(3), PP. 15-19, 2016.

[7] Devaraj S, Rosenson RS, Jialal I. Metabolic syndrome: an appraisal of the proinflammatory and procoagulant status. Endocrinol Metab Clin North Am,Vol.33, PP.431-453, 2004.

[8] A.D.Watson, M.Navab, S.YHama, A,Sevanian, Prescott SM et al. Effect of platelet activating factor-acetyl hydrolase on the formation and action of minimally oxidized low density lipoprotein. J Clin Invest,Vol.95(2), PP.774-782, 1995.

[9] D.K.Sanghera, N.Saha, C.E.Aston and M.Kamboh . Genetic polymorphism of paraoxonase and the risk of coronary heart disease. Arterioscler Thromb Vasc Biol,Vol.17(6), PP.1067-1073, 1997.

[10] Y.Okayama . Oxidative stress in allergic and inflammatory skin diseases .Curr Drug Targets: Inflamm Allergy,Vol. 4(4), PP. 517519, 2005

[11] Y.Yuan, V.Cruzat, P.Newshome, J.Cheng, Y.Chen, Regulation of SIRT1 in aging: roles in mitochondrial function and biogenesis. Mech Ageing Dev,vol.155, PP.10-21,2016.

[12] N.S.Nagane and J.V.Ganu. Lipid Profile and Serum Paraoxonase1 Activity in CRF Patients Pre and Post Hemodialysis. Al Ameen J Med Sci; ,Vol.4(1), PP.61-68, 2011.

[13] A.Ayub, M.I.Mackness, .Arrol, B.Mackness, J.Patel . Serum paraoxonase after myocardial infarction. Arterioscler Thromb Vasc Biol,Vol.19(2), PP.330-335, 1999. 
[14] M.A.Maged and M.Rushdy. Hyperlipidemia in association with pro-inflammatory cytokines among chronic spontaneous urticaria: case-control study. Eur Ann Allergy Clin Immunol,Vol.50(6), PP.254-261, 2018.

[15] A.Kasperska-Zajac, J.Sztylc, E.Machura and G. Jop. Plasma IL-6 concentration correlates with clinical disease activity and serum Creactive protein concentration in chronic urticaria patients. Clin Exp AllergyVol.41, PP.1386-1391, 2011.

[16] F.J.Pashkow. Oxidative stress and inflammation in heart disease: do antioxidants have a role in treatment and/or prevention?.Int J Inflam,Vol.22, PP.65-69,2011.
[17] M.Rajappa, L.Chandrashekar, I.Sundar, M.Munisamy, P.H.Ananthanarayanan. Platelet oxidative stress and systemic inflammation in chronic spontaneous urticaria. Clin Chem Lab Med ,Vol.51(9), PP.1789-1794, 2013.

[18] M.I.Mackness, D.Harty, D.Bhatnagar, P.H.Winocour, S.Arrol . Serum paraoxonase activity in familial hypercholesterolaemia and insulin-dependent diabetes mellitus. Atherosclerosis, Vol. 86(2), PP.193-199, 1991.

[19] S.M.Dyke, B.S.Carey and E.R.Kaminski . Effect of stress on basophil function in chronic idiopathic urticaria. Clin Exp Allergy,Vol.38(1), PP.86-92, 2008. 
Benha Journal Of Applied Sciences, Vol.(5) Issue(7) Part (1) (2020) 